\title{
Haptoglobin improves shock, lung injury, and survival in canine pneumonia
}

\author{
Kenneth E. Remy, ${ }^{1,2}$ Irene Cortés-Puch, ${ }^{1}$ Steven B. Solomon, ${ }^{1}$ Junfeng Sun, ${ }^{1}$ Benjamin M. Pockros, ${ }^{1}$ \\ Jing Feng, ${ }^{1}$ Juan J. Lertora, ${ }^{3}$ Roy R. Hantgan, ${ }^{4}$ Xiaohua Liu, ${ }^{5}$ Andreas Perlegas, ${ }^{5}$ H. Shaw Warren, ${ }^{6}$ \\ Mark T. Gladwin, ${ }^{7}$ Daniel B. Kim-Shapiro, ${ }^{5}$ Harvey G. Klein, ${ }^{8}$ and Charles Natanson ${ }^{1}$ \\ ${ }^{1}$ Critical Care Medicine Department, Clinical Center, NIH, Bethesda, Maryland, USA. ${ }^{2}$ Department of Pediatrics, Division of \\ Critical Care, Washington University in St. Louis, St. Louis, Missouri, USA. ${ }^{3}$ Clinical Pharmacology Program, Clinical Center, \\ $\mathrm{NIH}$, Bethesda, Maryland, USA. ${ }^{4}$ Department of Biochemistry, Wake Forest University Health Sciences, Winston-Salem, \\ North Carolina, USA. ${ }^{5}$ Department of Physics, Wake Forest University, Winston-Salem, North Carolina, USA. Infectious \\ Disease Unit, Massachusetts Ceneral Hospital, and Shriners Hospital for Crippled Children, Boston, Massachusetts, USA \\ ${ }^{7}$ Department of Medicine, The University of Pittsburgh, Pittsburgh, Pennsylvania, USA. ${ }^{8}$ Department of Transfusion \\ Medicine, Clinical Center, NIH, Bethesda, Maryland, USA.
}

Conflict of interest: MTG is a coinventor of pending patent applications and planned patents directed to the use of recombinant neuroglobin and heme-based molecules as antidotes for $\mathrm{CO}$ poisoning, which have recently been licensed by Globin Solutions Inc. (patent nos 8980871 and 9114109). MTC is a shareholder, advisor, and director in Globin Solutions Inc. Additionally, and unrelated to $\mathrm{CO}$ poisoning, MTC is a coinventor on patents directed to the use of nitrite salts in cardiovascular diseases, which have been licensed by United Therapeutics and Hope Pharmaceuticals, and is a coinvestigator in a research collaboration with Bayer Pharmaceuticals to evaluate riociguate as a treatment for patients with SCD. The work by the authors was done as part of US government-funded research however, the opinions expressed are not necessarily those of the NIH.

Submitted: June 21, 2018

Accepted: August 9, 2018

Published: September 20, 2018

Reference information:

JCI Insight. 2018;3(18):e123013.

https://doi.org/10.1172/jci.

insight.123013.
During the last half-century, numerous antiinflammatory agents were tested in dozens of clinical trials and have proven ineffective for treating septic shock. The observation in multiple studies that cell-free hemoglobin (CFH) levels are elevated during clinical sepsis and that the degree of increase correlates with higher mortality suggests an alternative approach. Human haptoglobin binds CFH with high affinity and, therefore, can potentially reduce iron availability and oxidative activity. CFH levels are elevated over approximately 24-48 hours in our antibiotic-treated canine model of $S$. aureus pneumonia that simulates the cardiovascular abnormalities of human septic shock. In this 96-hour model, resuscitative treatments, mechanical ventilation, sedation, and continuous care are translatable to management in human intensive care units. We found, in this $S$. aureus pneumonia model inducing septic shock, that commercial human haptoglobin concentrate infusions over 48-hours bind canine CFH, increase CFH clearance, and lower circulating iron. Over the 96-hour study, this treatment was associated with an improved metabolic profile ( $\mathrm{pH}$, lactate), less lung injury, reversal of shock, and increased survival. Haptoglobin binding compartmentalized CFH to the intravascular space. This observation, in combination with increasing CFHs clearance, reduced available iron as a potential source of bacterial nutrition while decreasing the ability for CFH and iron to cause extravascular oxidative tissue injury. In contrast, haptoglobin therapy had no measurable antiinflammatory effect on elevations in proinflammatory C-reactive protein and cytokine levels. Haptoglobin therapy enhances normal host defense mechanisms in contrast to previously studied antiinflammatory sepsis therapies, making it a biologically plausible novel approach to treat septic shock.

\section{Introduction}

Cell-free hemoglobin ( $\mathrm{CFH})$ is released into the plasma in conditions associated with $\mathrm{RBC}$ hemolysis including malaria, sickle cell disease, thalassemia, transfusion of stored RBCs, and extracorporeal circulation. Recently, CFH elevations have also been documented during bacterial sepsis (1-5). These increased plasma CFH levels have correlated with increased morbidity and mortality in preclinical and clinical studies $(3,5-10)$. Previous reports show that the release of $\mathrm{CFH}$ following stored-RBC transfusion is associated with increased mortality in canine models of $S$. aureus pneumonia, causing septic shock (11). The pathophysiology and mortality correlated with the presence and severity of pneumonia and the levels of both plasma CFH and plasma free iron. These findings were ameliorated during infection by washing the RBC concentrates to remove $\mathrm{CFH}$ and iron that accumulated during storage $(12,13)$.

The effects of intravascular hemolysis are postulated to be mediated by both direct and indirect mechanisms. Direct injury may be related to extravascular nitric oxide (NO) scavenging and reactive species formation by $\mathrm{CFH}$; subsequent vasoconstriction, causing ischemic endothelial damage $(1,2,10,14)$; or the release of heme and iron producing oxidative tissue injury (15-20). Proposed indirect mechanisms include 
heme-enhanced proinflammatory, innate immune responses damaging tissues $(21,22)$, and release of free iron, which worsens established infection by providing an essential nutrient that augments bacterial growth $(11,23)$. Additional support for the iron hypothesis has been supplied by studies using commercial i.v. iron preparations in a canine bacterial pneumonia model (24).

The normal low levels of CFH released during RBC turnover are rapidly cleared from the circulation through binding to haptoglobin, a naturally occurring plasma protein that forms high-affinity, high-molecular weight ( 150,000-400,000 Daltons) haptoglobin-hemoglobin complexes that are cleared by the reticuloendothelial system $(16,25)$. Clearance of haptoglobin-hemoglobin complexes during mild hemolysis is an efficient process. However, during conditions associated with excessive hemolysis, haptoglobin clearance can be saturated, resulting in circulation of $\mathrm{CFH}$, heme, and iron $(16,17,26)$. Large molecular weight haptoglobin-hemoglobin complexes can lead to rapid rises in intravascular levels and prolonged half-life of bound hemoglobin. The complexing also binds the free iron and heme in $\mathrm{CFH}$ and prevents these toxic products from extravasating into the extravascular space and causing injury.

We hypothesized that administration of pharmacologic doses of haptoglobin might reduce morbidity and mortality in our canine model of transfusion during sepsis. In a series of studies using a commercially fractionated plasma-derived haptoglobin concentrate, we found that excess haptoglobin improves outcomes in both transfused and, unexpectedly, nontransfused septic animals. These findings suggest that administration of haptoglobin may be an effective clinical therapy for managing sepsis and septic shock.

\section{Results}

Mortality. Animals with $S$. aureus pneumonia that were exchange-transfused and randomized to receive haptoglobin $(n=9)$ had a significantly decreased mortality rate compared with animals receiving no haptoglobin (septic controls receiving PBS, $n=9)(44 \%$ vs. $78 \%$ [ $43 \%$ relative decrease]; $P=0.03$ stratified logrank test) (Figure 1A). Animals with $S$. aureus pneumonia that were not exchange-transfused and randomized to receive haptoglobin $(n=12)$ vs. no haptoglobin (septic controls receiving $25 \%$ albumin, $n=12$ ) had a decreased mortality rate that approached statistical significance (50\% vs. $75 \%$ [ $33 \%$ relative decrease]; $P=$ 0.16 stratified log-rank test) (Figure 1B). Haptoglobin infusion during sepsis was associated with a similar beneficial effect with or without exchange-transfusion ( $43 \%$ vs. $33 \%$ relative decrease in mortality; $P=0.36$, for interaction, stratified Cox proportional hazards model).

Shock score. All animals were treated with vasopressors to normalize blood pressure. The shock score incorporates the amount of vasopressor support (norepinephrine) required to normalize the mean arterial pressure (13). Progressively positive and negative scores reflect more hypertension and hypotension, respectively. Overall, during $S$. aureus pneumonia, animals randomized to receive haptoglobin therapy had improved mean shock scores (more positive scores) compared with septic controls. In particular, for the septic animals undergoing RBC exchange transfusion, the beneficial effect of haptoglobin therapy with improved shock scores became statistically significant at 36, 48, and 72 hours, and for the septic animals without RBC exchange-transfusions, the effect was statistically significant at 36, 48, and 60 hours (Figure 1, C and D, respectively; Supplemental Figure 1; supplemental material available online with this article; https://doi.org/10.1172/jci.insight.123013DS1.

Lung injury score. The lung injury score (LIS) incorporates 5 variables of lung function (mean pulmonary artery pressure, alveolar arterial oxygen gradient, plateau pressure measurement, $\mathrm{SpO}_{2}$, and respiratory rate) to detect lung injury (13). Overall, during $S$. aureus pneumonia, animals randomized to receive haptoglobin infusion had improved mean LIS scores (lower scores indicating less lung injury) compared with septic controls. In particular, for the septic animals undergoing RBC exchange-transfusion, the beneficial effect of haptoglobin therapy became statistically significant at 36, 48, and 60 hours, while - for the septic animals that were not exchange-transfused - statistical significance was noted at 10, 24, 36, 48, 60, and 72 hours (Figure 1, E and F, and Supplemental Figure 2).

CFH. During sepsis with RBC exchange transfusion, mean plasma CFH levels were significantly elevated from baseline ( 0 hours) in animals randomized to receive haptoglobin therapy at $13,24,36$, and 48 hours and in septic controls (no haptoglobin) at 60 hours (Figure 2A). Mean combined bound hemoglobin-haptoglobin and $\mathrm{CFH}$ levels at 60 hours were significantly lower in septic-transfused animals receiving haptoglobin $(P=0.02)$ and were similar to baseline values $(P=0.84)$, whereas septic-transfused controls still had significantly elevated mean $\mathrm{CFH}$-alone levels at 60 hours compared with baseline. 
A
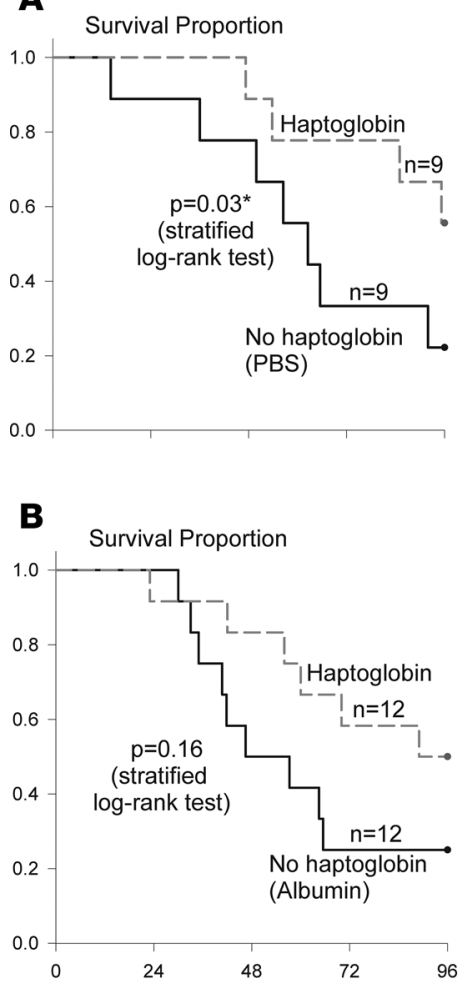

Sepsis with RBC Exchange-Transfusion

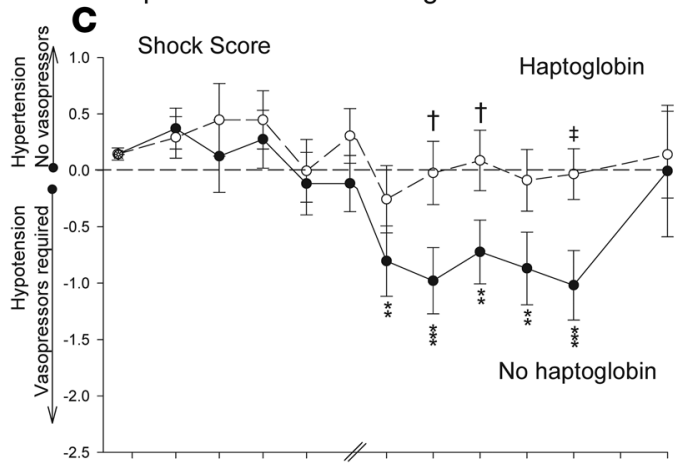

Sepsis without RBC Exchange-Transfusion

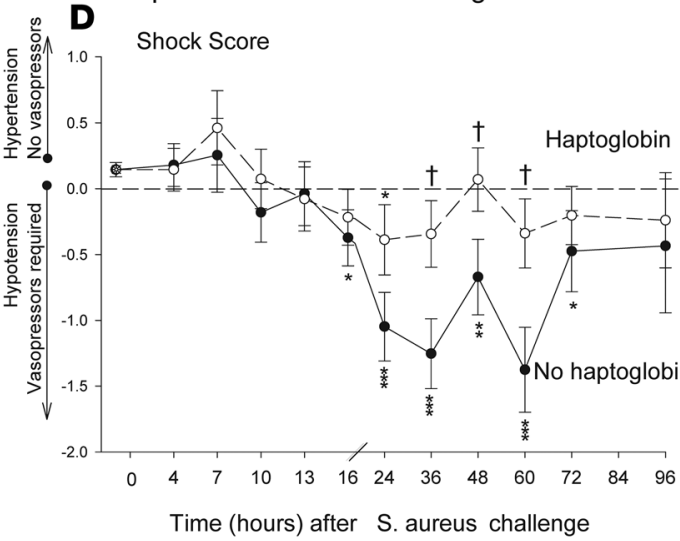

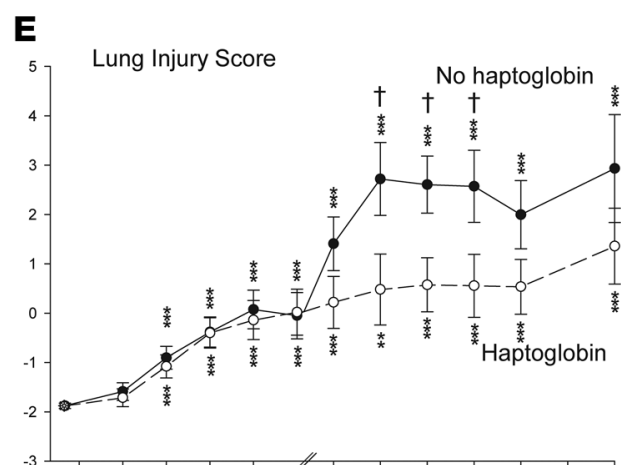

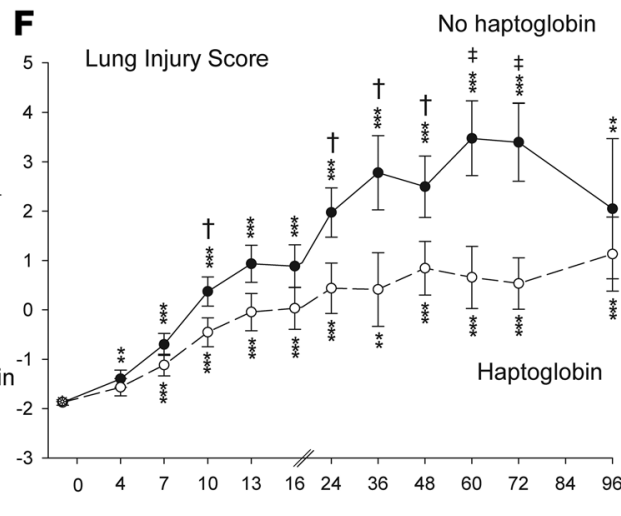

Change from time 0

Haptoglobin vs.

$\begin{array}{ll}* & >0.01 \text { and } \leq 0.05 \\ * & >0.001 \text { and } \leq 0.01\end{array}$

$\begin{array}{ll}* & >0.001 \\ * & \leq 0.001\end{array}$ No Haptoglobin

$\uparrow$ †

Figure 1. Kaplan-Meier survival curve for the 96-hour sepsis study. The survival comparison in canines receiving haptoglobin or no haptoglobin with (A) or without (B) RBC exchange transfusion after S. aureus challenge. $P$ values are denoted by asterisks indicating significance in comparison between each panel group using stratified log rank tests. (C and D) Mean shock scores ( \pm SEM) at serial time points. The shock score accounts for the level of vasopressor support (norepinephrine) needed to maintain the mean arterial pressure at a preset normal level for canines (mean 80 mmHg). Shock score is compared over 96 hours in canines receiving haptoglobin or no haptoglobin with (C) or without (D) RBC exchange transfusion. Changes from baseline are shown for each study group plotted from a common origin the mean value for animals at baseline. $P$ values indicate significance in each group comparison in each panel and are denoted by asterisks (for changes over time) or crosses (comparing haptoglobin vs. no haptoglobin at each time point). (E and F) Mean ( \pm SEM) lung injury scores (LIS) at serial time points. The LIS detects pulmonary damage via measurements in mean pulmonary artery pressure, alveolar-arterial oxygen gradient, plateau pressure, oxygen saturation, and respiratory rate. The LIS is plotted over time ( $x$ axis) for animals receiving haptoglobin or no haptoglobin with (E) or without (F) RBC exchange transfusion. Changes from baseline are shown for each study group plotted from a common origin, with the mean value for animals at baseline. $P$ values indicate significance in each group comparison in each panel and are denoted by asterisks (for changes over time) or crosses (comparing haptoglobin vs. no haptoglobin at each time point). Comparisons of all variables (except survival) were made based on contrasts in linear mixed models, which allow us to account for repeated measurements of each animal and the actual pairing of animals within each cycle.

During sepsis without exchange transfusion, mean plasma CFH levels were significantly elevated at 16 hours $(P=0.004)$ in animals receiving haptoglobin compared with baseline and were not significantly elevated at all other time points (Figure $2 \mathrm{~B}$ ). In septic controls without haptoglobin or exchange transfusion, $\mathrm{CFH}$ levels became elevated later, at 24 hours $(P=0.0002)$, and were not significantly elevated at all other time points (Figure 2B). Mean CFH levels at 24 hours were significantly lower in septic animals that were not exchange transfused receiving haptoglobin therapy $(P=0.01)$ and were similar to baseline values $(P=0.94)$, whereas septic controls still had significantly elevated CFH levels at 24 hours compared with baseline $(P=0.0002)$.

The CFH kinetics appear similar during sepsis with or without exchange transfusion, although - as expected - absolute levels are higher with RBC exchange. Specifically, haptoglobin therapy resulted in mean $\mathrm{CFH}$ increases earlier and more rapid clearance in both the exchange and no-exchange sepsis studies.

Nontransferrin bound iron (NTBI) levels. In animals with $S$. aureus pneumonia randomized to receive haptoglobin therapy and an exchange transfusion of RBCs, there were no significant increases in mean NTBI 


\section{Sepsis with RBC Exchange-Transfusion}
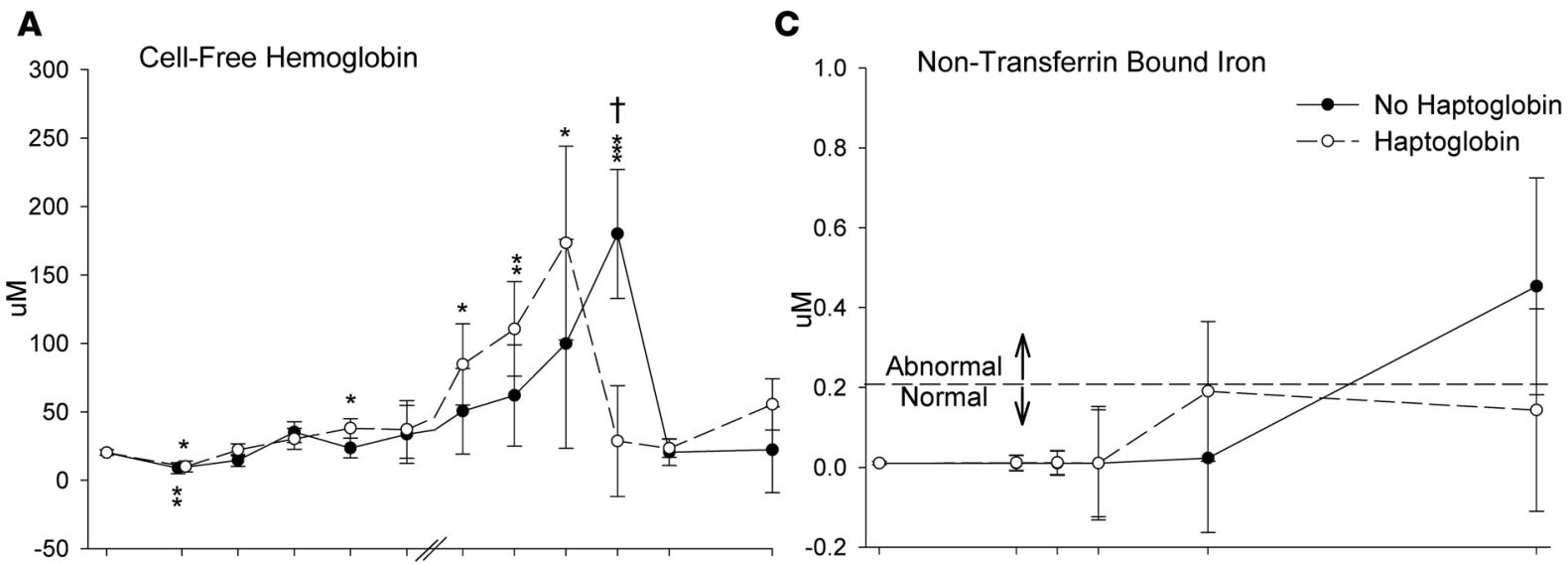

\section{Sepsis without RBC Exchange-Transfusion}
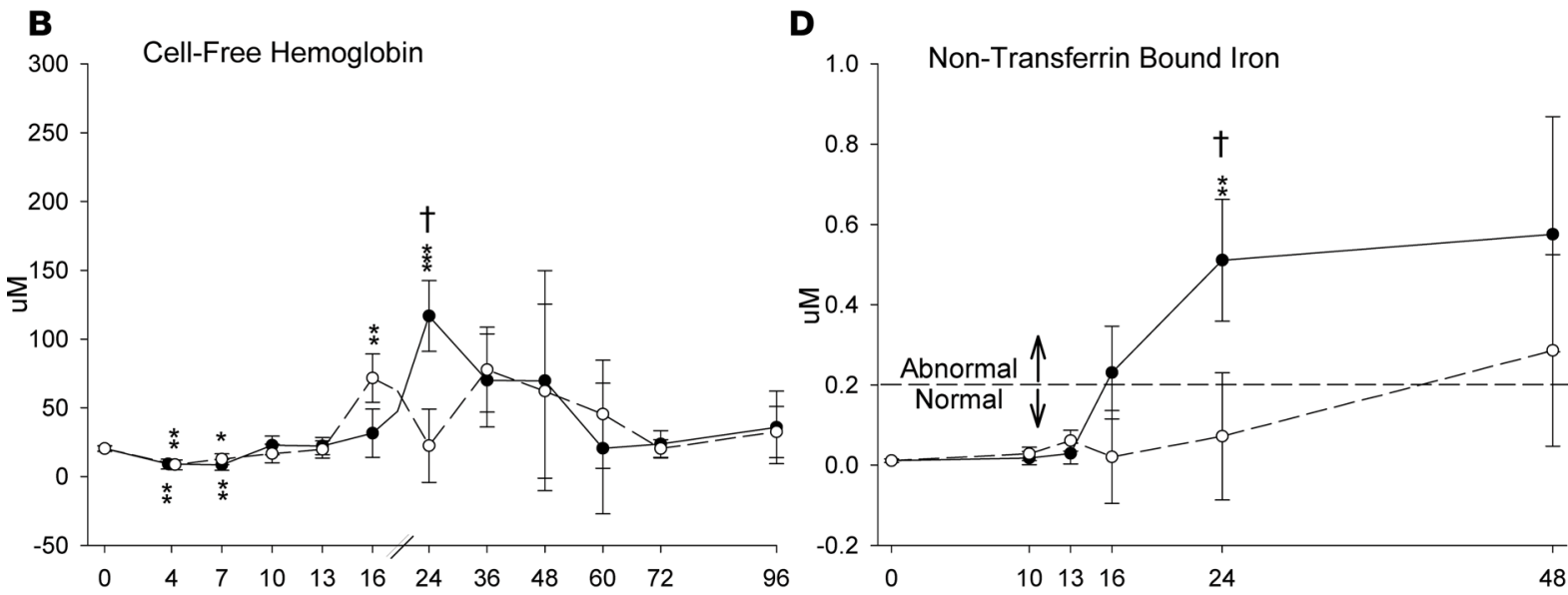

Time (hours) after S. aureus challenge

\begin{tabular}{clc}
$\begin{array}{c}\text { Change } \\
\text { from time } 0\end{array}$ & \multicolumn{2}{r}{$\begin{array}{c}\text { Haptoglobin v } \\
\text { No Haptoglobin }\end{array}$} \\
$*$ & $>0.01$ and $\leq 0.05$ & $\dagger$ \\
$*$ & $>0.001$ and $\leq 0.01$ & $\ddagger$ \\
$*$ & $\leq 0.001$ & $\ddagger$
\end{tabular}

$\mathbf{E}$

Binding of Haptoglobin to cell-free hemoglobin

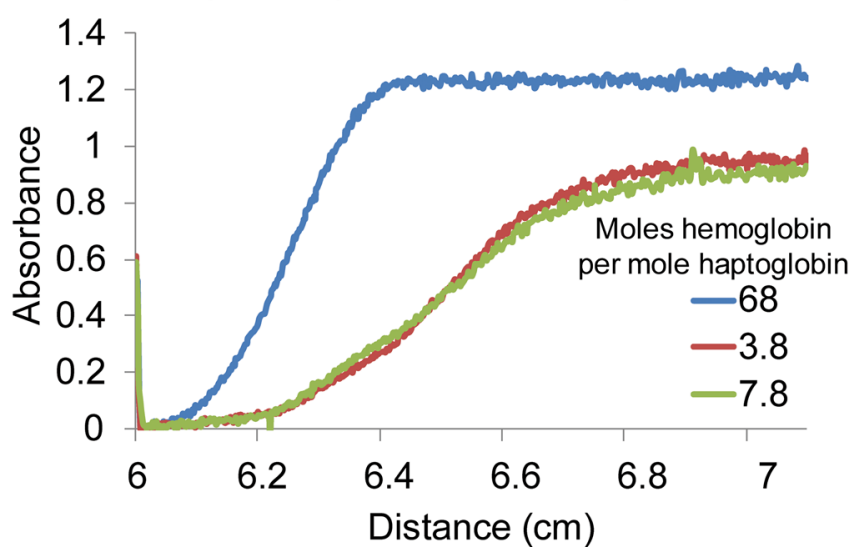

Figure 2. Mean cell-free hemoglobin, nontransferrin bound iron, and haptoglobin cell-free hemoglobin binding at serial time points. The format is similar to Figure 1, except that cell-free hemoglobin (A and B) and nontransferrin bound iron (C and $\mathbf{D}$ ) are shown. In addition, in panel $\mathbf{E}$, haptoglobin cell-free hemoglobin binding is shown. (A and B) The Drabkin's assay was used to measures both unbound and bound (with haptoglobin) cell-free hemoglobin. (E) Absorbance profile for mixtures of haptoglobin and hemoglobin. The samples were spun at $163,000 \mathrm{~g}$ at $25.2^{\circ} \mathrm{C}$, and absorbance was measured as a function of time and sedimentation distance. Absorbance was normalized to the maximum absorbance of all samples. The sample with a molar ratio of 68 to 1 of 
hemoglobin (in heme) to haptoglobin (in $\alpha$ - $\beta$ dimer), essentially CFH, sedimented much more slowly than the others. The other 2 samples with much greater ratios of hemoglobin to haptoglobin sedimented more rapidly, consistent with a higher molecular weight and confirming that the canine hemoglobin binds to human haptoglobin. Analysis of the sedimentation data provided the percentage of fast (bound) and slow (unbound) sediment species. That percentage determination, together with the known total concentrations of haptoglobin and hemoglobin in each sample, was used to calculate the binding stoichiometry. For example, when $227 \mu \mathrm{M}$ hemoglobin was mixed with $37 \mu \mathrm{M}$ haptoglobin, $75 \%$ of the total hemoglobin (170.25 $\mu \mathrm{M}$ ) was a slowly sedimenting species. Thus, $170.25 / 37=4.6$ hemoglobin molecules (in heme) per haptoglobin (in $\alpha-\beta$ dimers). Comparisons of all variables (except survival) were made based on contrasts in linear mixed models, which allow us to account for repeated measurements of each animal and the actual pairing of animals within each cycle.

levels at all time points studied - 10, 13, 16, 24, and 48 hours - compared with baseline (time 0), and the mean NTBI levels at all these time points were in the normal range $(<0.2 \mu \mathrm{M})$ (Figure $2 \mathrm{C})$. Septic controls with exchange transfusion (no haptoglobin) also had no significant increases in mean NTBI compared with baseline levels at 10,13,16, 24, and 48 hours; however, in septic controls (no haptoglobin), the mean levels of NTBI by 48 hours increased compared with baseline were trending to be elevated $(P=0.10)$ and reached the abnormal range $(>0.2 \mu \mathrm{M})$ (Figure $2 \mathrm{C}$ ).

In animals with $S$. aureus pneumonia and that were not exchange-transfusion randomized to receive haptoglobin therapy, there were no significant increases in mean NTBI levels at all time points studied $10,13,16,24$, and 48 hours - compared with baseline. In contrast, in septic controls (no haptoglobin), there were significant increases in mean NTBI levels compared with baseline at 24 hours $(P=0.001)$, with levels elevated and reaching the abnormal range $(>0.20 \mu \mathrm{M})$ at 16 hours $(P=0.060)$ and 48 hours $(P=$ 0.056). At 24 hours, mean NTBI levels in septic animals that were not exchange-transfused were significantly higher in no haptoglobin- compared with haptoglobin-receiving animals $(P=0.047)$ (Figure 2D). Haptoglobin therapy resulted in fewer increases in mean NTBI levels into the abnormal range during $S$. aureus pneumonia, independently of exchange-transfusing RBCs.

Human haptoglobin binding stoichiometry to canine $\mathrm{CFH}$. Ten samples with varying hemoglobin and haptoglobin concentrations were suspended in canine plasma, and the percentage of free hemoglobin that bound to haptoglobin was determined using the measured absorbance of hemoglobin at $415 \mathrm{~nm}$ as a function of time and sedimentation distance. Figure $2 \mathrm{E}$ shows the absorbance vs. sedimentation distance for 3 study samples with different molar ratios of hemoglobin to haptoglobin $(68,7.8$, and 3.8) taken 15 minutes after the beginning of sedimentation. The sample with 68 -fold more hemoglobin than haptoglobin, as expected, sedimented similar to $100 \%$ free, unbound hemoglobin (Figure $2 \mathrm{E}$ ). The other 2 samples sedimented more rapidly, consistent with a higher molecular weight and confirming that the canine hemoglobin binds to human haptoglobin. We found that, on average, there was $4.3 \pm 1.4$ hemoglobin molecules per haptoglobin, consistent with the expectation that each haptoglobin $\alpha-\beta$ dimer binds 2 hemoglobin dimers ( 4 heme).

Human haptoglobin levels. In animals with $S$. aureus pneumonia randomized to receive haptoglobin therapy vs. septic controls (no haptoglobin), whether exchange-transfused or not, there were significant increases in mean human haptoglobin levels at all time points studied: 4-96 hours (Figure 3, A and B). The constancy of the levels over these 96 hours suggests that we reached and maintained a steady state with our dosing regimen ( 2 doses of $100 \mathrm{mg} / \mathrm{kg}$, doses given 3 hours apart, followed by $12.5 \mathrm{mg} / \mathrm{kg} / \mathrm{hour}$ infusion until the 48-hour termination point). However, in animals with $S$. aureus pneumonia not transfused vs. transfused, mean haptoglobin levels were greater during the time of (4-48 hours) exchange transfusion, and this reached statistical significance at 24 hours (i.e., mean haptoglobin levels in the treatment group at 24 hours vs. controls [no haptoglobin] levels were significantly increased more without vs. with transfusion [interaction, $P=0.04])$. In $S$. aureus pneumonia animals that were not exchange-transfused, greater mean haptoglobin level increases were associated with greater mean central venous pressure (CVP) levels and lower mean hematocrit levels at multiple time points. Mean CVP in the septic haptoglobin treatment group vs. septic controls (no haptoglobin) were significantly greater without vs. with exchange-transfusion at 13 and 48 hours (interaction), and mean hematocrit levels in the septic haptoglobin treatment group vs. septic controls (no haptoglobin) were significantly lower without vs. with exchange transfusion at 4 and 10 hours (interaction; Figure 3, C-F). The $S$. aureus pneumonia animals without exchange transfusion, thus, had higher mean haptoglobin levels, resulting in increased intravascular volume, increased CVP, and lower hemoglobin concentrations. The differential in haptoglobin levels and potential oncotic pressure effects cannot easily explain the similar beneficial effects seen with haptoglobin therapy in this study in septic animals with and without exchange transfusion, since animals with $S$. aureus pneumonia that were exchange-transfused had no significant differences in mean CVP with haptoglobin therapy compared with septic controls (no haptoglobin). There were also no significant differences in cardiac filling pressures as 
A Sepsis with RBC Exchange-Transfusion
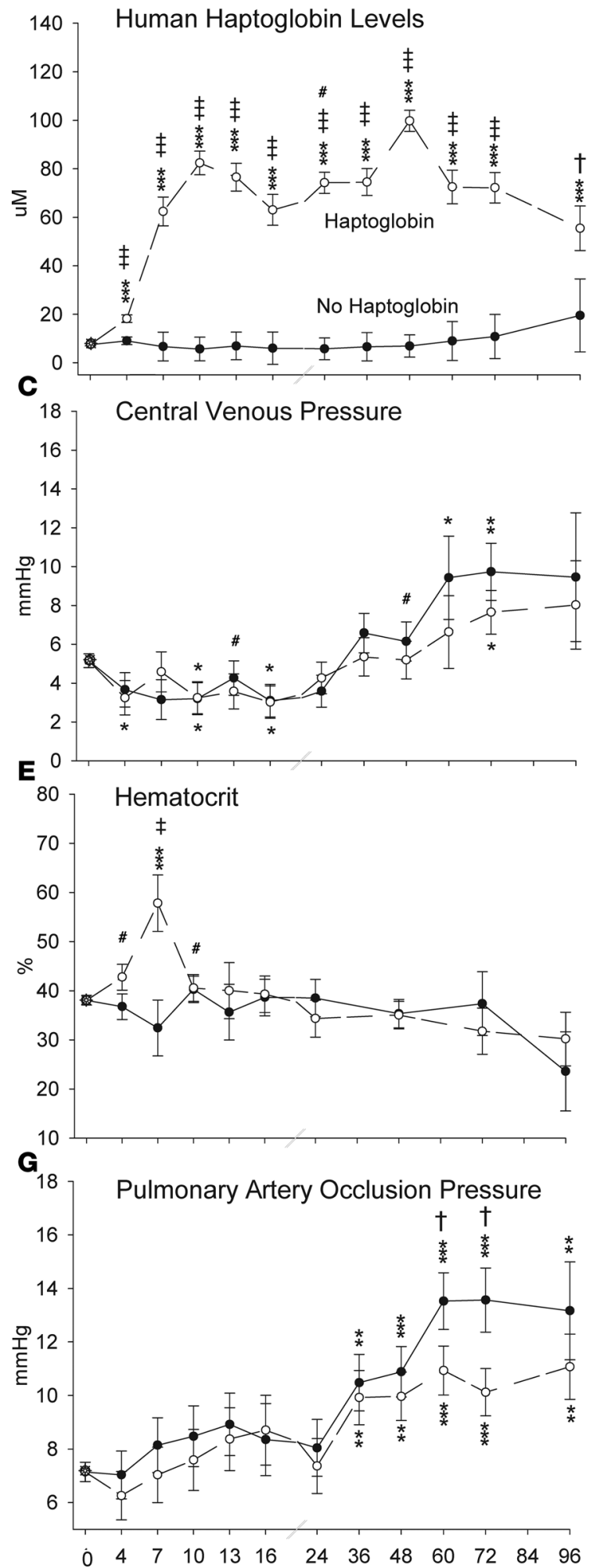

B Sepsis without RBC Exchange-Transfusion

Human Haptoglobin Levels

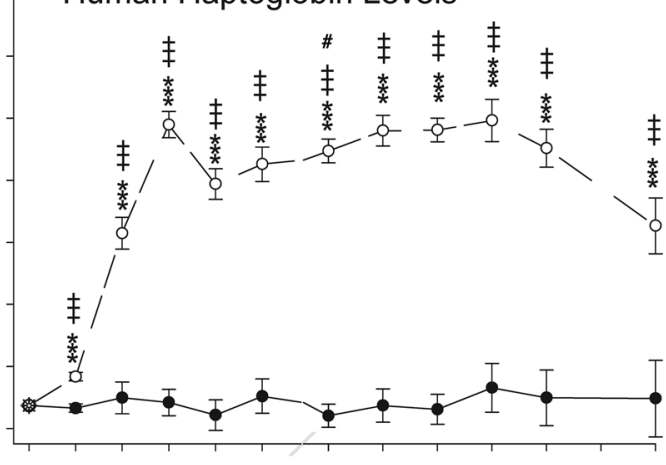

D

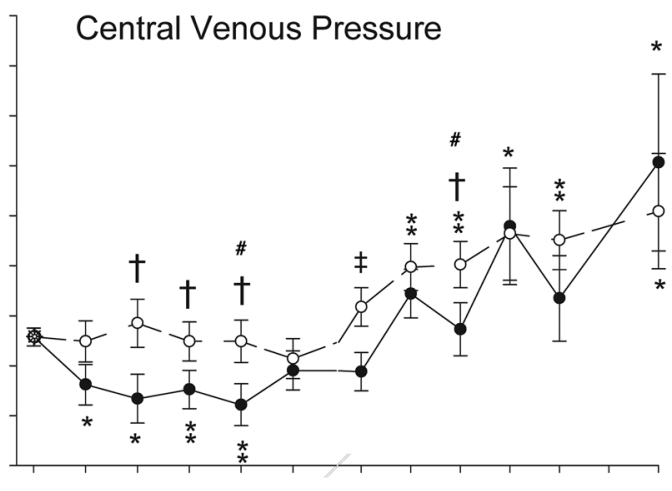

$\mathbf{F}$

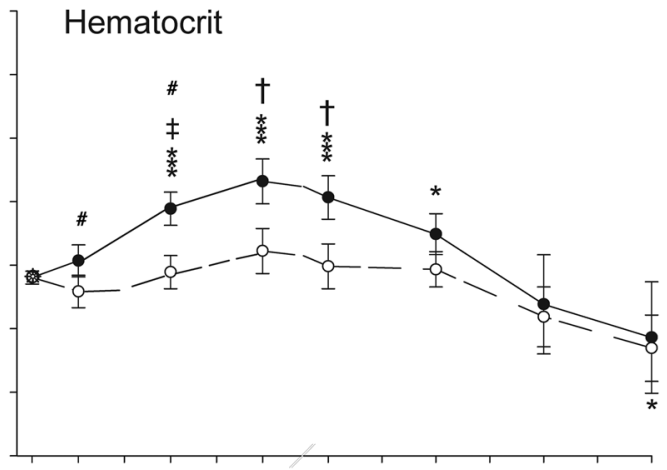

H

Pulmonary Artery Occlusion Pressure

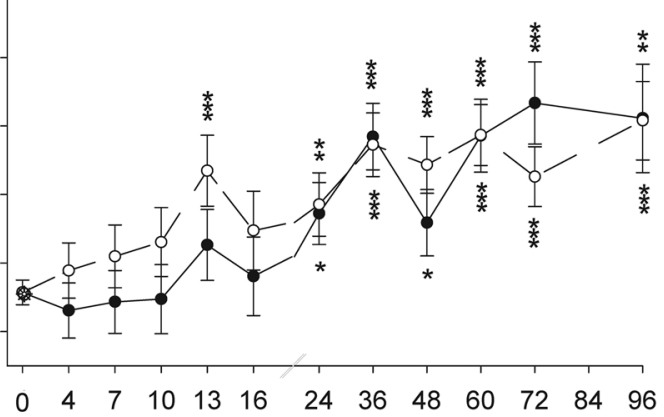

Time (hours) after $\mathrm{S}$. aureus Challenge

$\begin{array}{ll}\frac{\begin{array}{c}\text { Change } \\ \text { from time } 0\end{array}}{\frac{\text { Haptoglobin vs. }}{\text { No Haptoglobin }}} \\ *>0.01 \text { and } \leq 0.05 & \dagger>0.01 \text { and } \leq 0.05 \\ * \quad>0.001 \text { and } \leq 0.01 & \neq>0.001 \text { and } \leq 0.01 \\ * \leq 0.001 & \neq \leq 0.001\end{array}$

\# The effect of Haptoglobin vs. No Haptoglobin therapy is significantly different in the sepsis study with RBC Exchange-Transfusion compared to without $(p<0.05)$ 
Figure 3. Human haptoglobin, central venous pressure, hematocrit, and pulmonary artery occlusion pressure measurements at serial time points. The format is similar to Figure 1, except that human haptoglobin, central venous pressure, hematocrit, and pulmonary artery occlusion pressure are measured over 96 hours after $\mathbf{S}$. aureus challenge in canines receiving haptoglobin or no haptoglobin with $(\mathbf{A}, \mathbf{C}, \mathbf{E}, \mathbf{G})$ or without RBC exchange transfusion (B, D, F, H) Comparisons of all variables (except survival) were made based on contrasts in linear mixed models, which allow us to account for repeated measurements of each animal and the actual pairing of animals within each cycle.

measured by mean pulmonary artery occlusion pressure (PAOP) in animals with $S$. aureus pneumonia that were exchange-transfused and treated with haptoglobin compared with septic controls (no haptoglobin) over the first 48 hours (Figure 3G). However, there were significant increases in mean PAOP at 60 and 72 hours compared with baseline in $S$. aureus animals that were exchange-transfused but only among septic controls (no haptoglobin) compared with haptoglobin-treated septic animals (Figure 3D). Finally, there were also no significant differences in mean PAOP pressures in $S$. aureus pneumonia animals that did not receive an exchange transfusion of $\mathrm{RBC}$ between haptoglobin-treated animals vs. septic controls (no haptoglobin) throughout the experiment (Figure $3 \mathrm{H}$ ).

Metabolic function. Animals with $S$. aureus pneumonia that were exchange-transfused and randomized to receive haptoglobin therapy had significantly smaller decreases in mean arterial $\mathrm{pH}$ at 48 and 60 hours compared with septic controls (Figure 4A). This apparent beneficial effect of haptoglobin therapy on acid-base balance (i.e., $\mathrm{pH}$ ) during sepsis with exchange transfusion was also associated with an overall improved metabolic profile as follows: During sepsis with exchange transfusion, haptoglobin therapy resulted in significantly smaller increases in mean partial pressure of $\mathrm{CO}_{2}\left(\mathrm{pCO}_{2}\right)$ at 48 and 60 hours compared with septic controls (Figure 4C), as well as significantly smaller decreases in base deficit at 60 hours $(P=0.008)$ (Figure 4E) and significantly lower mean serum lactate levels at 7, 13, 24 and 48 hours (Figure 4G).

Animals with $S$. aureus pneumonia that were exchange-transfused but not randomized to receive haptoglobin therapy had significantly smaller decreases in mean arterial $\mathrm{pH}$ at 36 and 60 hours compared with septic controls (Figure 4B). This beneficial effect of haptoglobin therapy on $\mathrm{pH}$ was associated with an overall improved metabolic profile as follows: Septic controls had a significantly increased mean $\mathrm{pCO}_{2}$ at 24 and 60 hours compared with baseline, whereas there were no significant increases in mean $\mathrm{pCO}_{2}$ from baseline at all time points studied in septic animals treated with haptoglobin (Figure 4D). Haptoglobin therapy resulted in smaller increases of the mean base deficit at all time points studied that reached statistical significance at 96 hours $(P=0.04)$ (Figure $4 \mathrm{~F})$. Lastly, in septic controls, there was a significant increase in serum mean lactate level at 13 and 36 hours compared with baseline, whereas among septic animals with haptoglobin therapy, no significant increases in mean lactate levels compared with baseline were found throughout (Figure $4 \mathrm{H}$ ).

$C$-reactive protein, $T N F-\alpha, I L-6$, and $I L-10$. Animals with $S$. aureus pneumonia randomized to receive haptoglobin therapy and septic controls had no significant changes from baseline at 4, 16, and 24 hours in mean TNF- $\alpha$, IL-6, and IL-10 levels in either model studied. There were also no significant difference comparing the effect of haptoglobin therapy vs. septic controls, except for in the sepsis-only model. The decrease from baseline in mean IL-10 levels with haptoglobin therapy at 4, 16, and 24 hours - although nonsignificant compared with baseline - was a significantly greater decrease compared with septic controls at all these time points (Supplemental Table 5). There were marked similar increases (pg/ml) from baseline in mean (SEM) C-reactive protein levels with haptoglobin therapy and in septic controls in both models at 24 hours (no transfusion: $18.6 \pm 0.8 \mathrm{pg} / \mathrm{ml}$ at baseline and $19.3 \pm 0.8 \mathrm{pg} / \mathrm{ml}$ at 24 hours; transfusion: $16.7 \pm 0.9$ at baseline and $17.5 \pm 0.9$ at 24 hours) and 48 hours (no transfusion; $19.1 \pm 0.8 \mathrm{pg} / \mathrm{ml}$ and $19.6 \pm 0.9 \mathrm{pg} /$ $\mathrm{ml}$; and transfusion: $18.2 \pm 0.9 \mathrm{pg} / \mathrm{ml}$ and $19.0 \pm 0.9 \mathrm{pg} / \mathrm{ml}$, respectively; all, $P<0.0001)$. However, these elevations were not significantly different between haptoglobin therapy and septic controls at 24 hours or 48 hours in either model studied $(P>0.40)$. Thus, we found no evidence of an antiinflammatory effect of haptoglobin therapy in this study during sepsis or sepsis with RBC transfusion.

Renal, hepatic, and cardiac function; electrolytes; glucose; platelets; transferrin bound iron levels; and white blood cells. Animals with $S$. aureus pneumonia randomized to receive haptoglobin therapy vs. septic controls had some differences in mean values for renal (creatinine and blood urea nitrogen; BUN), hepatic (alanine aminotransferase; ALT), aspartate aminotransferase (AST), lactate dehydrogenase (LDH), and total bilirubin (Supplemental Table 1) and cardiac function (cardiac output by thermodilution technique [V24/26, Philips Medical]) and left ventricular ejection fraction as measured by cardiac echocardiography (Sonos 5500, Philips Medical) (Supplemental Table 2), electrolytes (sodium, potassium, and chloride), and glucose 
A Sepsis with RBC Exchange-Transfusion
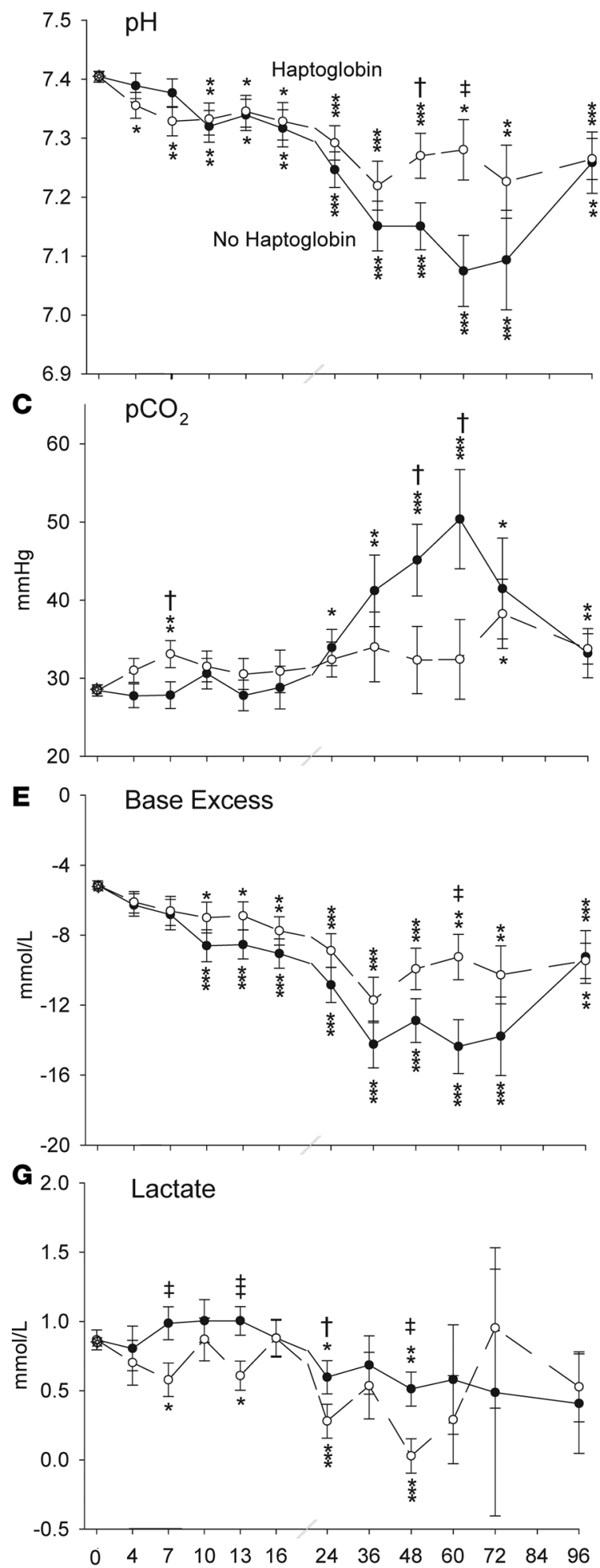

B Sepsis without RBC Exchange-Transfusion
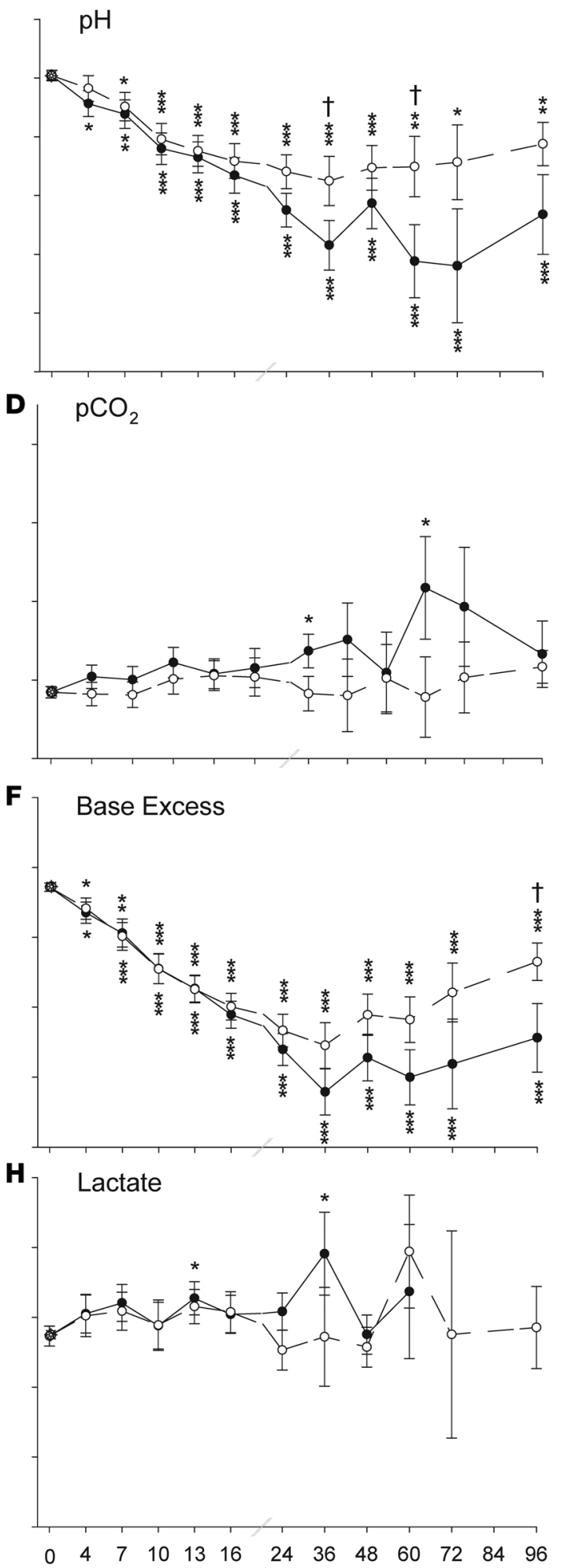

Time (hours) after $\mathrm{S}$. aureus Challenge

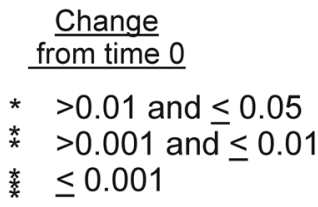

Change

* $>0.001$ and $\leq 0.01$

崣 $\leq 0.001$
Haptoglobin vs.

No Haptoglobin

$\dagger>0.01$ and $\leq 0.05$

$\neq>0.001$ and $\leq 0.01$

丰 $\leq 0.001$ 
Figure 4. Arterial blood gas comparison over the 96-hour duration of the sepsis study. The format is similar to Figure 1, except that quantitative arterial blood gas measurements $\left(\mathrm{pH}, \mathrm{pCO}_{2}\right.$, base excess) and lactate levels are compared over 96 hours after $\mathrm{S}$. aureus challenge in canines receiving haptoglobin or no haptoglobin with $(\mathbf{A}, \mathbf{C}, \mathbf{E}, \mathbf{G})$ or without RBC exchange-transfusion (B, D, F, H). Comparisons of all variables (except survival) were made based on contrasts in linear mixed models, which allow us to account for repeated measurements of each animal and the actual pairing of animals within each cycle.

(Supplemental Table 3), as well as platelet counts and transferrin-bound iron levels (Supplemental Table 4) and circulating white blood cells (Supplemental Figure 3) from 4-96 hours in one sepsis model or the other. However, these mostly random time point differences in one model or the other cannot easily explain the consistent beneficial effects of haptoglobin therapy in this study with and without RBC exchange transfusion on shock, LIS, and survival.

\section{Discussion}

Over the last half-century, numerous agents tested in sepsis trials, enrolling tens of thousands of patients, have failed to show reproducible benefits (27-30). Most of these failed therapeutic approaches were attempts to decrease inflammation during sepsis by inhibiting host responses to bacteria. Here, in randomized controlled trials in our canine model of lethal $S$. aureus pneumonia that was originally designed to study the effect of haptoglobin administration on exchange transfusion, marked elevations were demonstrated in plasma $\mathrm{CFH}$ levels during sepsis, even in the absence of blood transfusion. Haptoglobin therapy bound $\mathrm{CFH}$ and decreased $\mathrm{CFH}$ and iron levels associated with an improved metabolic profile ( $\mathrm{pH}$, lactate levels), less lung injury, decreased severity of shock, and improved survival rates. We posit that haptoglobin binds increased levels of $\mathrm{CFH}$ in sepsis, compartmentalizes these bound complexes to the intravascular space, and promotes $\mathrm{CFH}$ clearance, decreasing plasma iron - an essential nutrient for bacteria - and preventing extravascular oxidative injury from $\mathrm{CFH}$ and iron. As a result, haptoglobin appears to be an effective treatment and may offer a promising alternative strategy to treat sepsis because of its ability to enhance natural host mechanisms (16), in contrast to previous approaches designed to inhibit them (30). Plasma-derived human haptoglobin has been approved in Japan since 1985 for multiple clinical indications associated with elevated CFH levels, including hemolytic anemias, and for use during extracorporeal circulation (31).

Haptoglobin infusions were associated in both our sepsis models with earlier spikes in CFH levels, which is indirect evidence that haptoglobin successfully compartmentalized the bound complexes to the intravascular space. Using analytical centrifugation techniques, we confirmed human haptoglobin was binding canine $\mathrm{CFH}$. The large haptoglobin-CFH complexes formed would be greatly confined to the intravascular space until metabolized through specific receptors by reticuloendothelial cells, unlike free $\mathrm{CFH}$ molecules, which dissociates into dimers that pass into the urine and extravasate into the extravascular space. We measured $\mathrm{CFH}$ using an assay that measures free $\mathrm{CFH}$ in the plasma, as well as bound$\mathrm{CFH}$. As haptoglobin binds $\mathrm{CFH}$, the resultant complexes are confined to the intravascular circulation, decreasing the volume of distribution of CFH and causing an earlier spike in Drabkin's-measured CFH levels compared with studies without haptoglobin. In septic canines with or without RBC exchange transfusion, haptoglobin produced earlier rises in $\mathrm{CFH}$ levels and accelerated $\mathrm{CFH}$ clearance, which resulted in free iron levels that were overall lower and within the normal ranges. This compartmentalization of $\mathrm{CFH}$ has the added potential benefit during sepsis of decreasing the ability for $\mathrm{CFH}$ and iron to cause extravascular oxidative tissue injury.

Studies in normal guinea pigs and mice have previously reported that haptoglobin therapy can ameliorate the direct nephrotoxic and lethal effects of elevated CFH from stored blood $(6,8,32)$. A study in mice found that administering hemopexin, another naturally occurring protein that binds heme released by $\mathrm{CFH}$, prevented tissue damage and decreased mortality during cecal-ligation and puncture (CLP) (21). Other murine studies employing CLP to induce sepsis found that genetic disruption of haptoglobin expression enhances mortality and administering haptoglobin concentrates improves mortality in WT and mice genetically deficient for haptoglobin (33).

In previous studies, we found that the combination of transfusing RBCs stored for 6 weeks with increasing dose challenges of $S$. aureus progressively worsened the dose-dependent effects of septic shock (12). While the elevated levels of CFH remained relatively stable across bacterial doses, the free iron levels progressively decreased in a dose-dependent fashion (12). This observation is consistent with the notion that, as the bacterial dose increased, $S$. aureus scavenged and utilized the iron released with hemolysis, which likely promoted bacterial growth and worsened septic shock. In healthy canines 
without infection, transfusing stored RBCs with elevated $\mathrm{CFH}$ levels is nontoxic (12), producing none of the nephrotoxic or lethal effects seen in healthy guinea pigs and mice $(6,8,32)$. In hemorrhagic shock models in canines without infection, resuscitation with stored RBCs with high CFH levels improved the hemodynamic response to hemorrhage reperfusion (34). These findings lead us to conclude that iron from $\mathrm{CFH}$ is a relevant if not critical factor during bacterial infection and can explain, in large part, why elevation in $\mathrm{CFH}$ worsens the condition of septic canines and why increases in $\mathrm{CFH}$ are associated with increased mortality in humans, especially those at risk for or with an established infection (3-5). Haptoglobin infusion, by binding and increasing clearance of $\mathrm{CFH}$, could promote nutritional immunity by isolating the iron from bacteria, inhibiting bacterial growth, and curbing the effects of bacterial infections. Haptoglobin compartmentalizing $\mathrm{CFH}$ to the intravascular spaces may also reduce tissue injury by excluding $\mathrm{CFH}$ from extravascular sites of $\mathrm{NO}$ production and from NO effector sites, preventing NO depletion and ischemic injury $(35,36)$. In addition, binding the heme molecule in $\mathrm{CFH}$ could also reduce proinflammatory injury in the lung $(15,16,22)$ and direct oxidative lung injury $(16,17)$. Haptoglobin could also directly bind proinflammatory mediators and improve outcomes (33). However, we found no evidence that haptoglobin infusions directly affect proinflammatory mediators such as C-reactive protein, which are released during sepsis.

The beneficial effects of haptoglobin therapy in our sepsis studies without RBC transfusion were biochemically and physiologically less evident compared with sepsis with transfusion, and the survival benefit was smaller ( $33 \%$ vs. $43 \%$ relative decrease in mortality), despite studying more animals $(n=$ 24 vs. $n=18$ ). Conversely, the haptoglobin effect on lung injury was greater without RBC transfusion, with statistically significant differences at many more time points $(10,24,36,48,60$, and 72 hours vs. 36,48 , and 60 hours). This apparently contradictory observation may reflect the presence of 2 separate haptoglobin effects on survival, one dependent on $\mathrm{CFH}$ clearance and the second independent of $\mathrm{CFH}$ but related to interspecies anaphylaxis. During our pretrial dose-finding studies, we found that human haptoglobin can induce anaphylaxis in canines in a dose-dependent fashion (Supplemental Methods). The higher haptoglobin levels at earlier time points in the sepsis without exchange-transfusion animals, due to the absence of the wash out that occurs with exchange transfusion, may have triggered immune activation sufficient to worsen the survival outcome of the bacterial infection. Cross species anaphylaxis would not be an issue for clinical trials. Human serum albumin can also bind heme, albeit weakly $(32,37)$. Albumin binding may have reduced the relative beneficial survival effect of haptoglobin binding of $\mathrm{CFH}$. Using PBS instead of albumin as a control solution during the RBC exchange transfusion trial avoided this possibility, but it does not easily explain the contradictory effect on lung injury $(32,37)$.

In our animal model, a sudden bolus of bacteria is given to an otherwise healthy animal. Human bacterial infection likely begins with a small nidus of infection and progresses slowly over time, often due to some impairment in host defenses. These differences may make our experimental model results less applicable to the human condition. Furthermore, our results could vary with a different bacterial species, dose challenge, site of infection, or experimental species. Lastly, the model cannot fully reproduce all the treatments and heterogeneity in human infections, which could make the beneficial effects seen here nonreproducible in humans.

In conclusion, our studies demonstrate that haptoglobin binds $\mathrm{CFH}$, compartmentalizes the molecule into the intravascular space, quickly clears it from the circulation, and decreases the amount of available intravascular iron. Bacteria that cause human infections are known to produce hemolysins, a virulence factor that disrupts $\mathrm{RBCs}$ and releases $\mathrm{CFH}$ into the circulation $(38,39)$. Elevated levels of $\mathrm{CFH}$ have been described with clinical sepsis, and higher levels have been correlated with increased mortality (3-5). We found that binding and clearing of $\mathrm{CFH}$ during bacterial infection was associated with an improved metabolic profile ( $\mathrm{pH}$, lactate levels), decreases in shock score, and lung injury - all findings consistent with and supporting our demonstrated improvement in sepsis mortality rates. Our haptoglobin hypothesis has a theoretically better risk-benefit profile than previous therapeutic approaches inhibiting inflammation tested for bacterial sepsis due to the ability of haptoglobin to enhance a normal host defense mechanism (16), rather than inhibiting it (30). These findings support a role for pharmacologic infusions of haptoglobin as a biologically plausible new approach to bacterial sepsis for testing in human sepsis trials. 


\section{Methods}

Supplemental Methods are available online with this article.

In the first of 2 sepsis experiments, 24 purpose-bred beagles (18-30 months old, 9-2.5 kg, Covance Inc.) with $S$. aureus pneumonia were randomized to receive i.v. human haptoglobin (2-1 and 2-2) plasma fraction concentrates (a gift from CSL Bearing) $(800 \mathrm{mg} / \mathrm{kg}$ total dose, in 2 divided $100 \mathrm{mg} / \mathrm{kg}$ bolus doses at 4 and 7 hours after infection followed immediately by a $600 \mathrm{mg} / \mathrm{kg}$ continuous infusion over 48 hours) or an osmotically equivalent volume of human $25 \%$ albumin (Talecris Biotherapeutics; Supplemental Figure 4). This model has been shown to reproduce the cardiovascular abnormalities of human septic shock (40). To create conditions translatable to the clinical intensive care unit environment, all animals received fluids, vasopressors, mechanical ventilation, and sedation titrated to physiologic endpoints, as well as oxacillin (Auromedics LLC) $30 \mathrm{mg} / \mathrm{kg}$ every 4 hours starting 4 hours after bacterial challenge for 96 hours $(11,41)$. All animals were treated identically, except for the experimental intervention, and - if alive after 96 hours - were considered survivors and euthanized.

In a second sepsis experiment, 18 purpose-bred beagles (18-30 months old, 9-12.5 kg) with experimental $S$. aureus pneumonia were additionally exchanged-transfused with $80 \mathrm{ml} / \mathrm{kg}$ of 7 -day-old stored canine universal donor blood (DEA 1.1 ABRINT) in 4 divided doses $(20 \mathrm{ml} / \mathrm{kg}$ ) given sequentially every 3 hours starting 4 hours after infection but otherwise treated the same and given haptoglobin therapy as above (Supplemental Figure 1). Albumin was not used in the second experiment as a control solution to avoid binding heme, which is expected to increase after hemolysis of transfused RBCs (32, 37). The PBS cohort in the second experiment serves as a vehicle control. A detailed description of the experimental model and procedures has been published previously $(11,41)$. See Supplemental Methods for details of haptoglobin administration, desensitization protocol, and binding studies, as well as details of other laboratory procedures performed and the statistical methods.

Statistics. Survival times were plotted using Kaplan-Meier survival curves and analyzed using stratified log-rank tests and stratified Cox proportional hazards models. For all other variables, changes from baseline values were analyzed. To evaluate shock reversal, we standardized the mean arterial pressure (MAP) and norepinephrine (NE) concentration using Z-scores and then calculated a shock reversal score based on the difference of their respective $\mathrm{Z}$ scores (higher score indicates improvement in shock reversal). To evaluate pulmonary function, we constructed a LIS (lower score indicates less lung injury) based on the first principal component of mean pulmonary artery pressure, arterial alveolar oxygen gradient, plateau pressures, breathing rates, and a pulse oximeter oxygen saturation measurement $\left(\mathrm{SpO}_{2}\right)$. Linear mixed models were used to account for repeated measurements of each animal and the actual pairing of animals within each cycle. SAS version 9.3 was used for all analyses. All p-values are 2-tailed and considered significant if $\mathrm{p} \leq 0.05$.

Study approval. All experiments were conducted after protocol approval (no. CCM13-14) by the Animal Care and Use Committee of the Clinical Center at the NIH.

\section{Author contributions}

KER, ICP, SBS, and HGK designed the study, performed experiments, provided data analysis and figure generation, and wrote/edited the manuscript; JS performed all statistical analysis; BMP, JF, XL, AP, and RRH performed relevant experiments; JJL provided pharmacokinetics support for experimental design; HSW, MTG, and DBKS performed relevant experiments and analysis, and edited the manuscript; CN designed the study, supervised experiments, analyzed and interpreted the data, and wrote/edited the manuscript. All authors reviewed and approved the final version of the manuscript.

\section{Acknowledgments}

The authors would like to acknowledge Juli Maltagliati and Kelly Byrne for their assistance with manuscript and figure preparation. This work was supported by Intramural NIH National Heart, Lung and Blood Institute funds; NIH National Heart, Lung and Blood Institute external grants 2R01HL098032, 1R01HL125886, 5P01HL103455, and T32 HL110849; the Institute for Transfusion Medicine Hemophilia Center of Western Pennsylvania; and the Shriners Hospital for Crippled Children (grant no. 8600). The work by the authors was done as part of US government-funded research; however, the opinions expressed are not necessarily those of the NIH. 
Address correspondence to: Charles Natanson, National Institutes of Health, Critical Care Medicine Department, Building 10, Room 2C136, Bethesda, Maryland 20892, USA. Phone: 301.496.4335; Email: cnatanson@cc.nih.gov.

1. Gladwin MT, Kim-Shapiro DB. Storage lesion in banked blood due to hemolysis-dependent disruption of nitric oxide homeostasis. Curr Opin Hematol. 2009;16(6):515-523.

2. Reiter CD, et al. Cell-free hemoglobin limits nitric oxide bioavailability in sickle-cell disease. Nat Med. 2002;8(12):1383-1389.

3. Janz DR, et al. Association between cell-free hemoglobin, acetaminophen, and mortality in patients with sepsis: an observational study. Crit Care Med. 2013;41(3):784-790.

4. Janz DR, et al. Association between haptoglobin, hemopexin and mortality in adults with sepsis. Crit Care. 2013;17(6):R272.

5. Adamzik M, Hamburger T, Petrat F, Peters J, de Groot H, Hartmann M. Free hemoglobin concentration in severe sepsis: methods of measurement and prediction of outcome. Crit Care. 2012;16(4):R125.

6. Baek JH, et al. Hemoglobin-driven pathophysiology is an in vivo consequence of the red blood cell storage lesion that can be attenuated in guinea pigs by haptoglobin therapy. J Clin Invest. 2012;122(4):1444-1458.

7. Remy KE, Natanson C, Klein HG. The influence of the storage lesion(s) on pediatric red cell transfusion. Curr Opin Pediatr. 2015;27(3):277-285.

8. Baek JH, Zhang X, Williams MC, Schaer DJ, Buehler PW, D'Agnillo F. Extracellular Hb enhances cardiac toxicity in endotoxemic guinea pigs: protective role of haptoglobin. Toxins (Basel). 2014;6(4):1244-1259.

9. Billings FT, Ball SK, Roberts LJ, Pretorius M. Postoperative acute kidney injury is associated with hemoglobinemia and an enhanced oxidative stress response. Free Radic Biol Med. 2011;50(11):1480-1487.

10. Meyer C, et al. Hemodialysis-induced release of hemoglobin limits nitric oxide bioavailability and impairs vascular function. J Am Coll Cardiol. 2010;55(5):454-459.

11. Solomon SB, et al. Mortality increases after massive exchange transfusion with older stored blood in canines with experimental pneumonia. Blood. 2013;121(9):1663-1672.

12. Wang D, et al. Transfusion of older stored blood worsens outcomes in canines depending on the presence and severity of pneumonia. Transfusion. 2014;54(7):1712-1724

13. Cortés-Puch I, et al. Washing older blood units before transfusion reduces plasma iron and improves outcomes in experimental canine pneumonia. Blood. 2014;123(9):1403-1411.

14. Donadee $\mathrm{C}$, et al. Nitric oxide scavenging by red blood cell microparticles and cell-free hemoglobin as a mechanism for the red cell storage lesion. Circulation. 2011;124(4):465-476.

15. Fortes GB, et al. Heme induces programmed necrosis on macrophages through autocrine TNF and ROS production. Blood. 2012;119(10):2368-2375.

16. Schaer DJ, Vinchi F, Ingoglia G, Tolosano E, Buehler PW. Haptoglobin, hemopexin, and related defense pathways-basic science, clinical perspectives, and drug development. Front Physiol. 2014;5:415.

17. Schaer DJ, Buehler PW, Alayash AI, Belcher JD, Vercellotti GM. Hemolysis and free hemoglobin revisited: exploring hemoglobin and hemin scavengers as a novel class of therapeutic proteins. Blood. 2013;121(8):1276-1284.

18. Mollan TL, Alayash AI. Redox reactions of hemoglobin: mechanisms of toxicity and control. Antioxid Redox Signal. 2013;18(17):2251-2253

19. Remy KE, et al. Mechanisms of red blood cell transfusion-related immunomodulation. Transfusion. 2018;58(3):804-815.

20. Alayash AI. Redox biology of blood. Antioxid Redox Signal. 2004;6(6):941-943.

21. Larsen R, et al. A central role for free heme in the pathogenesis of severe sepsis. Sci Transl Med. 2010;2(51):51ra71.

22. Graça-Souza AV, Arruda MA, de Freitas MS, Barja-Fidalgo C, Oliveira PL. Neutrophil activation by heme: implications for inflammatory processes. Blood. 2002;99(11):4160-4165.

23. Hod EA, et al. Transfusion of red blood cells after prolonged storage produces harmful effects that are mediated by iron and inflammation. Blood. 2010;115(21):4284-4292.

24. Suffredini DA, et al. Parenteral irons versus transfused red blood cells for treatment of anemia during canine experimental bacterial pneumonia. Transfusion. 2017;57(10):2338-2347.

25. Kristiansen M, et al. Identification of the haemoglobin scavenger receptor. Nature. 2001;409(6817):198-201.

26. Nielsen MJ, Moestrup SK. Receptor targeting of hemoglobin mediated by the haptoglobins: roles beyond heme scavenging. Blood. 2009;114(4):764-771.

27. Deans KJ, Haley M, Natanson C, Eichacker PQ, Minneci PC. Novel therapies for sepsis: a review. J Trauma. 2005;58(4):867-874

28. Minneci PC, Deans KJ, Banks SM, Eichacker PQ, Natanson C. Meta-analysis: the effect of steroids on survival and shock during sepsis depends on the dose. Ann Intern Med. 2004;141(1):47-56.

29. Sweeney DA, Danner RL, Eichacker PQ, Natanson C. Once is not enough: clinical trials in sepsis. Intensive Care Med. 2008;34(11):1955-1960.

30. Eichacker PQ, et al. Risk and the efficacy of antiinflammatory agents: retrospective and confirmatory studies of sepsis. Am J Respir Crit Care Med. 2002;166(9):1197-1205.

31. Tanaka K, et al. Administration of haptoglobin during cardiopulmonary bypass surgery. ASAIO Trans. 1991;37(3):M482-M483.

32. Graw JA, et al. Haptoglobin or Hemopexin Therapy Prevents Acute Adverse Effects of Resuscitation After Prolonged Storage of Red Cells. Circulation. 2016;134(13):945-960.

33. Yang H, et al. Identification of CD163 as an antiinflammatory receptor for HMGB1-haptoglobin complexes. JCI Insight. 2016;1(7):e85375.

34. Solomon SB, et al. Transfused older stored red blood cells improve the clinical course and outcome in a canine lethal hemorrhage and reperfusion model. Transfusion. 2015;55(11):2552-2563.

35. Azarov I, et al. Rate of nitric oxide scavenging by hemoglobin bound to haptoglobin. Nitric Oxide. 2008;18(4):296-302. 
36. Wang D, et al. In vivo reduction of cell-free methemoglobin to oxyhemoglobin results in vasoconstriction in canines. Transfusion. 2013;53(12):3149-3163.

37. Satoh T, Satoh H, Iwahara S, Hrkal Z, Peyton DH, Muller-Eberhard U. Roles of heme iron-coordinating histidine residues of human hemopexin expressed in baculovirus-infected insect cells. Proc Natl Acad Sci USA. 1994;91(18):8423-8427.

38. Craven RR, et al. Staphylococcus aureus alpha-hemolysin activates the NLRP3-inflammasome in human and mouse monocytic cells. PLoS ONE. 2009;4(10):e7446.

39. Bubeck Wardenburg J, Patel RJ, Schneewind O. Surface proteins and exotoxins are required for the pathogenesis of Staphylococcus aureus pneumonia. Infect Immun. 2007;75(2):1040-1044.

40. Natanson C, Fink MP, Ballantyne HK, MacVittie TJ, Conklin JJ, Parrillo JE. Gram-negative bacteremia produces both severe systolic and diastolic cardiac dysfunction in a canine model that simulates human septic shock. J Clin Invest. 1986;78(1):259-270.

41. Minneci PC, et al. A canine model of septic shock: balancing animal welfare and scientific relevance. Am J Physiol Heart Circ Physiol. 2007;293(4):H2487-H2500. 